\title{
Internet des Objets (IdO) : Concepts, Enjeux, Défis et
}

\section{Perspectives}

\section{Internet of Things (IoT): Concepts, Issues, Challenges and Perspectives}

\author{
Imad Saleh ${ }^{1}$ \\ ${ }^{1}$ Laboratoire Paragraphe, Université Paris 8, imad.saleh@univ-paris8.fr
}

\begin{abstract}
RÉSUMÉ. Cet article est un approfondissement de notre article1 qui a été publié en 2017 dans la revue. Nous avons pris certains éléments mais nous avons également développé d'autres concepts pour faire une synthèse conséquente sur I'IdO. Nous présentons, 1) Objet connecté (OC), 2) Internet des objets : Définition, 3) Étapes et technologies dans l'écosystème de l'IdO, 4) IdO vers l'internet of Everything (loE), 5) IdO et Big Data, 6) Cloud computing appliqué au Big Data et à I'IdO, 8) Data Science et IdO, 9) Les enjeux et les défis de I'IdO, 10) Opportunités et menaces dans l'écosystème de l'IdO, 11) Sécurité de l'IdO, 12) Blockchain et IdO, et 13) en conclusion, nous faisons une synthèse sur les perspectives de l'Ido.

ABSTRACT. This article is a deepening of our article that was published in 2017 in the journal. We took some elements but we also developed other concepts to make a consequent synthesis on the loT. We present, 1) Connected Object (OC), 2) Internet of Things: Definition, 3) Steps and Technologies in the loT Ecosystem, 4) loT to the Internet of Everything (loE), 5) loT and Big Data, 6) Cloud computing applied to Big Data and IoT, 8) Data Science and loT, 9) Issues and challenges of loT, 10) Opportunities and threats of the loT ecosystem, 11) Security of the loT, 12) Blockchain and IoT, and 13) in conclusion, we summarize the perspectives of Ido.

MOTS-CLÉS. Objet connecté, internet des objets (IdO), internet of Everything (loE), interopérabilité, Big Data, Ecosystème de l'IdO, data science, trace, données privées, données publiques, éthique, Blockchain.

KEYWORDS. Connected object, Internet of Things (IOT), internet of Everything (IoE), interoperability, big data, data science, cloud computing, trace, private data, Information science, ethics, Blockchain.
\end{abstract}

\section{Introduction}

Internet en général et le web en particulier n'ont cessé d'évoluer : du web des informations au web des objets ${ }^{2}$ individualisés, via divers objets connectés grâce à la miniaturisation et le développement technologique, qui permet un double aspect : être connecté et communicant en permanence sans contrainte pour l'espace et le temps afin de répondre aux exigences et aux besoins de l'usager au niveau des services, de la communication et de l'information [ROX 2017, THE 2013].

Internet se transforme progressivement en un HyperRéseau, comme un réseau formé par des multitudes de connexions entre des Artefacts (physiques, documentaires), des acteurs (biologiques, algorithmiques), des écritures et des concepts (linked data, metadata, ontologies, folksonomie), appelé «Internet of Things (IoT) Internet des objets (IdO)», connectant des milliards d'êtres humains mais aussi des milliards d'objets. Il devient l'outil le plus puissant jamais inventé par l'homme pour créer, modifier et partager les informations. Cette transformation montre l'évolution du réseau d'internet: d'un réseau de calculateurs vers un réseau d'ordinateurs personnels, puis vers un réseau nomade intégrant les technologies des communications [CHA 2012]. Les développements des technologies Machine-to-Machine (M2M) pour le contrôle de machine à distance et aussi l'apparition dans l'année

\footnotetext{
${ }^{1}$ Saleh, I., 2017. « Les enjeux et les défis de I'Internet des Objets (IdO) », revue Internet des objets 1. DOI:10.21494/ISTE.OP.2017.0133

${ }^{2}$ En 2011 Vlad Trifa développe le concept de «Web des Objets » (WdO) dans sa thèse, c'est l'intégration des objets connectés dans le réseau Internet ainsi que dans le Web. Ce concept est basé sur le couplage des « webs social, programmable, sémantique, physique et temps réel, autant de facettes particulières que possèderait le WdO " [ROX 2017, p. 38].

Vlad Mihai Trifa, "Building blocks for a participatory Web of things » (Diss., Eidgenössische Technische Hochschule ETH Zürich, Nr. 19890, 2011, 2011), http://e-collection.library.ethz.ch/view/eth:4641. 
2000 d'IP (Internet Protocole) sur les réseaux mobiles cellulaires ont accéléré l'évolution de M2M vers l'IdO [WOO 2011].

\section{Objet connecté (OC)}

Avant de définir les concepts d'IdO, il est important de définir l'objet connecté qui est un dispositif dont la finalité première n'est pas d'être un système informatique ni une interface d'accès au web, exemple, un objet tel qu'une machine à café ou une serrure était conçue sans intégration de systèmes informatiques ni connexion à Internet. L'intégration d'une connexion Internet a un OC permet de l'enrichir en terme de fonctionnalité, d'interaction avec son environnement, il devient un OC Enrichi (OCE), par exemple, l'intégration d'une connexion internet à la machine à café la rendant accessible à distance.

Un OC peut interagir avec le monde physique de manière indépendante sans intervention humaine. Il possède plusieurs contraintes telles que la mémoire, la bande passante ou la consommation d'énergie, etc. Il doit être adopté à un usage, il a une certaine forme d'intelligence, une capacité de recevoir, de transmettre des données avec des logiciels grâce aux capteurs embarqués [ROX 2017]. Un objet connecté a une valeur lorsqu'il est connecté à d'autres objets et briques logicielles, par exemple : une montre connectée n'a d'intérêt qu'au sein d'un écosystème orienté santé/bien-être, qui va bien audelà de connaître l'heure.

Un OC à trois éléments clés :

- Les données produites ou reçues, stockées ou transmises.

- Les algorithmes pour traiter ces données.

- L'écosystème dans lequel il va réagir et s'intégrer.

Les propriétés d'usage d'un OC [SAL 2017]:

- Ergonomie (utilisabilité, maniabilité, ...).

- Esthétisme (formes/couleurs/sons/sensations, ...).

- Usage (histoire culturelle, profil, matrice sociale, ...).

- Méta-Morphisme (adaptabilité, personnalisation, modulation, ...).

Certains chercheurs parlent des " hyper objets » [MAV 2003] capables de mutualiser leurs ressources afin d'effectuer des tâches du quotidien, ils sont reliés par des «liens invisibles » au sein d'un même écosystème. Dans ce contexte, certains chercheurs comme [WEI 1993] ont déjà envisagé de l'informatique ubiquitaire là où «les technologies les plus profondes sont celles qui sont devenues invisibles. Celles qui, nouées ensemble, forment le tissu de notre vie quotidienne au point d'en devenir indissociables » [WEI 1991, p. 94].

La communication entre les objets passés par des identifications connues entre eux. Un objet doit avoir un ou plusieurs identifiants (codes-barres) pour être reconnu par un autre et établir la connection. Le système GS1 (mettre la référence) a proposé une technologie basée sur des étiquettes RFID ${ }^{3}$ associant de manière unique, les informations logistiques liées à un objet, à une adresse URL. Google a proposé le projet Physical Web pour associer de manière unique une adresse URL à un objet ${ }^{4}$. L'omniprésence dans notre vie des objets hétérogènes, mobiles et fragiles pose le problème des modèles de confiance adaptés à cet écosystème complexe et fragile [SZO 2017] ? Derrière ces

\footnotetext{
${ }^{3}$ RFID est un procédé de radio-identification s'apparentant au code-barres.

${ }^{4}$ https://google.github.io/physical-web/

() 2018 ISTE OpenScience - Published by ISTE Ltd. London, UK - openscience.fr
} 
technologies apparaissent la bataille pour les normes et standards pour 1'IdO entre les entreprises géantes d'internet car chacune souhaite imposer ses technologies.

\section{Internet des objets : Définition}

Kevin Ahston ${ }^{5}$, le cofondateur de l'Auto-ID Center du MIT a employé le terme «Internet Of Things (Internet des Objets ) » en 1999. IdO a été prononcé dans le cadre d'une présentation pour l'entreprise Procter \& Gamble (P\&G). Ce terme convoque, le monde d'objets, d'appareils et de capteurs qui sont interconnectés ${ }^{6}$ par internet.

Le CERP-IdO "Cluster des projets européens de recherche sur l'Internet des objets » définit l'Internet des objets comme : "une infrastructure dynamique d'un réseau global. Ce réseau global a des capacités d'auto-configuration basée sur des standards et des protocoles de communication interopérables. Dans ce réseau, les objets physiques et virtuels ont des identités, des attributs physiques, des personnalités virtuelles et des interfaces intelligentes, et ils sont intégrés au réseau d'une façon transparente » [CLU 2010].

Cette définition montre les deux aspects de l'IdO : temporel et spatial qui permettent aux personnes de se connecter de n'importe où à n'importe quel moment à travers des objets connectés [CHA 2012] (Figure 1) (Smartphone, tablettes, capteurs, caméras de vidéosurveillance, etc.). L'Internet des objets doit être pensé pour un usage facile et une manipulation sécurisée pour éviter des menaces et risques potentiels, tout en masquant la complexité technologique sous-jacente.

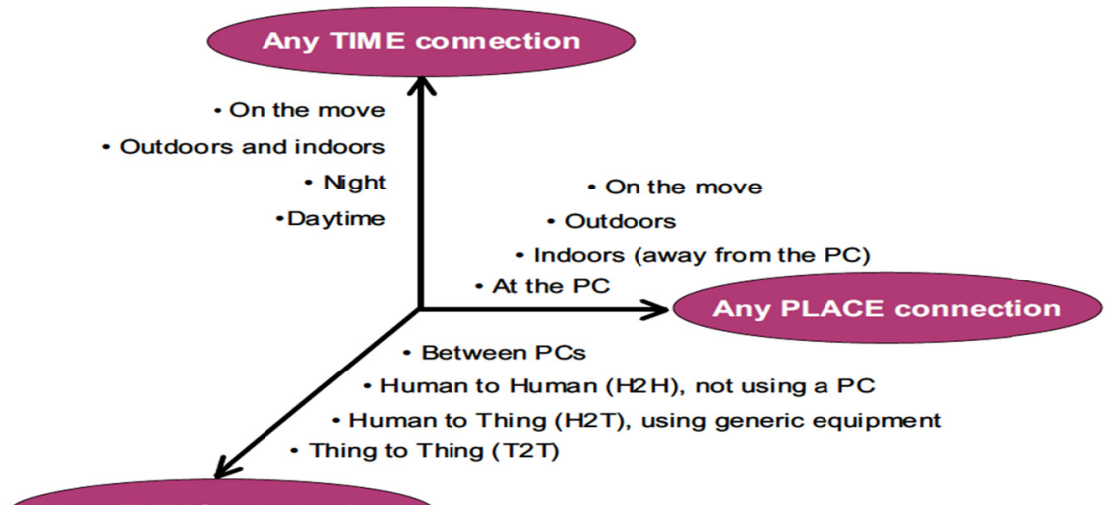

Any THING connection

Figure 1. Une nouvelle dimension pour I'IdO (Source ITU 2005 [INT 2005] tirée de [CHA 2012]).

L'évolution rapide de cet «Internet des Objets » bouleverse les frontières entre l'ordinateur et les produits du quotidien, cela est dû à deux facteurs : la généralisation des ressources informatiques et l'appropriation des services Web par les utilisateurs [THE 2013].

\subsection{Applications}

Les applications de l'IdO touchent pratiquement aujourd'hui toute la vie quotidienne :

- La santé et les systèmes de télésurveillance pour aider les personnes.

- L'agriculture connectée pour optimiser l'usage de l'eau.

- Les véhicules connectés aident à optimiser la gestion du trafic urbain.

\footnotetext{
${ }^{5}$ Il a participé à la création du standard RFID

6 "That “Internet of Things" Thing - RFID Journal », consulté le 2 janvier 2017, http://www.rfidjournal.com/articles/view?4986.

(C) 2018 ISTE OpenScience - Published by ISTE Ltd. London, UK - openscience.fr Page $\mid 3$
} 
- Les appareils électroménagers connectés aident à optimiser la consommation et la distribution de l'énergie électrique.

- Les arts numériques.

- Les montres connectées pour le bien être et le sport.

- Etc.

Ces exemples d'applications montrent que l'IdO est intégré dans notre vie quotidienne de plus, il améliore la qualité de vie des personnes [BOU 2017, NOY 2017, AMR 2017, GAG 2017, CRO 2017]. Il engendre un nouveau marché en créant de nouveaux emplois et métiers. Il aide également à la croissance pour les entreprises, et donne un élan pour la compétitivité. Selon la GSMA [GSM], l'IdO est une industrie en pleine expansion à tous les niveaux matériels et logiciels qui devrait apporter aux opérateurs mobiles un revenu confortable autour de \$1200 milliards vers 2020.

\section{4. Étapes et technologies dans l'écosystème de l'IdO}

Les OC sont au cour de l'IdO, mais il est important de pouvoir connecter l'ensemble de ces objets, les faire échanger des informations et interagir, au sein d'un même environnement. La mise en place de l'IdO passe par les étapes suivants : l'identification, l'installation de capteurs, la connexion des objets entre eux, l'intégration et la connexion à un réseau. Le Tableau 1 présente lles étapes et les protocoles éventuels [ROX 2017].

\begin{tabular}{|c|c|c|c|c|}
\hline Identifier & Capter & Connecter & Intégrer & $\begin{array}{c}\text { Mettre en } \\
\text { réseaux }\end{array}$ \\
\hline $\begin{array}{c}\text { Rendre possible } \\
\text { 1'identification } \\
\text { de chaque } \\
\text { élément } \\
\text { connecté. }\end{array}$ & $\begin{array}{c}\text { Mise en place de } \\
\text { dispositifs nous } \\
\text { rapprochant du } \\
\text { monde réel. Les } \\
\text { fonctions de base des } \\
\text { objets (le capteur de } \\
\text { température pour le } \\
\text { thermomètre par } \\
\text { exemple). }\end{array}$ & $\begin{array}{c}\text { Établir une } \\
\text { connexion } \\
\text { entre tous les } \\
\text { objets afin } \\
\text { dialoguer et } \\
\text { s'échanger des } \\
\text { données. }\end{array}$ & $\begin{array}{c}\text { Disposer d'un } \\
\text { moyen de } \\
\text { communication } \\
\text { rattachant les } \\
\text { objets au monde } \\
\text { virtuel. }\end{array}$ & $\begin{array}{c}\text { Relier les objets } \\
\text { et leurs données } \\
\text { au monde } \\
\text { informatique via } \\
\text { un réseau } \\
\text { (Internet par } \\
\text { exemple). }\end{array}$ \\
$\begin{array}{c}\text { IPv4, IPv6, } \\
\text { 6LoWPAN }\end{array}$ & $\begin{array}{c}\text { MEMS, RF MEMS, } \\
\text { NEMS }\end{array}$ & SigFox, LoRa & $\begin{array}{c}\text { RFID, NFC, } \\
\text { Bluetooth, } \\
\text { Bluetooth LE, } \\
\text { ZigBee, WiFi, } \\
\text { réseaux } \\
\text { cellulaires }\end{array}$ & $\begin{array}{c}\text { AllJoyn, REST } \\
\text { CoAP, MQTT, }\end{array}$ \\
\hline
\end{tabular}

Tableau 1. Les étapes et les technologies pour la mise en place de l'ldO [tiré de ROX 2017, p. 73]

\subsection{Architecture de I'ldO}

$\mathrm{Vu}$ le développement rapide de l'IdO, il devenait nécessaire d'avoir une architecture de référence qui permettrait d'uniformiser la conception des systèmes et favoriserait 1' interopérabilité7 et la communication entre les différents écosystèmes de 1'IdO (la figure 2 présente la chaine de valeur IdO/ $\mathrm{M} 2 \mathrm{M}$ ). Par exemple, un objet de marque $\mathrm{X}$ devra pouvoir envoyer des informations à une plateforme $\mathrm{Y}$ via le réseau Z. L'interopérabilité peut être vue sous deux angles, soit "fermée" au sein de grands écosystèmes qui partagent les mêmes standards, soit "native" basée sur des standards plus globaux, exemple, la v1 de l'internet avec IP ou HTTP.

\footnotetext{
${ }^{7}$ Voir les articles de Frédéric Charles dans le journal Zdnet : http://www.zdnet.fr/blogs/green-sii/iot-sortir-de-l-internet-des-silos39855298.htm 


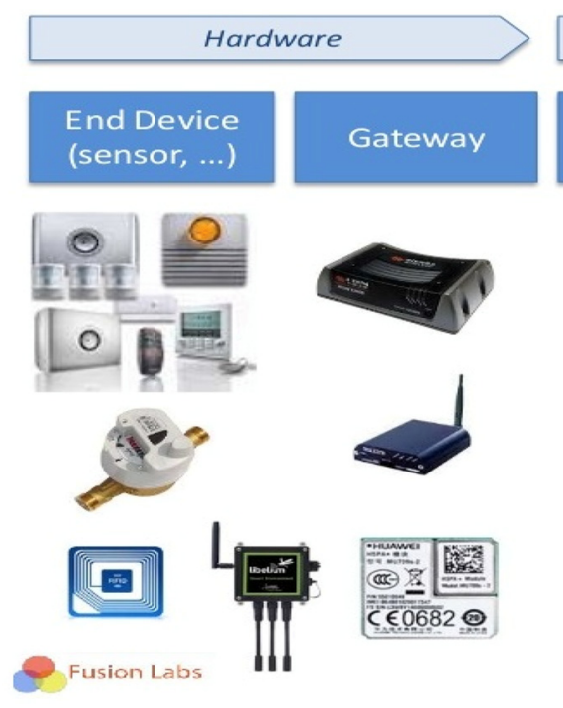

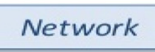

Network

connectivity

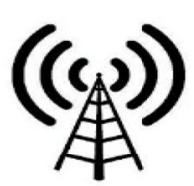

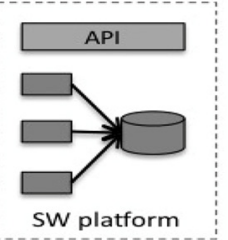
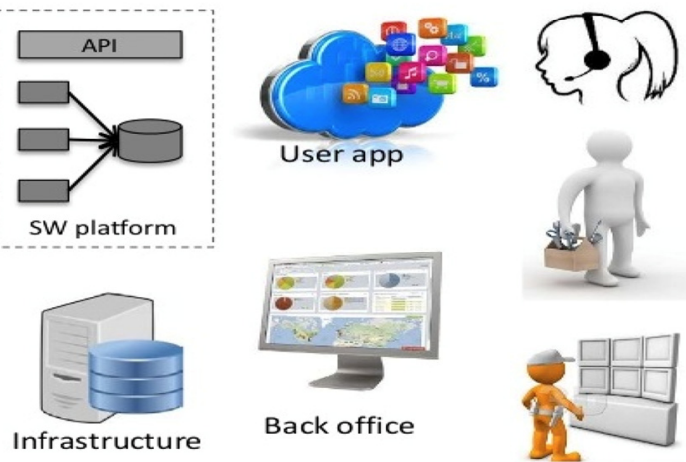

Figure 2. Tirée de la présentation de Stéphane Monteil (Janvier 2016)8

En mars 2015, le comité Internet Architecture Board (IAB) 9 édite la RFC10 7452. Il propose quatre modèles communs d'interactions entre des acteurs de l'IdO'11 [voir ROX 2017, p. 64] :

- La communication entre objets, ce modèle est basé sur une communication sans-fil entre deux objets. Les informations sont transmises grâce à l'intégration d'une technologie de communication sansfil comme ZigBee ou Bluetooth, etc.

- La communication des objets vers le cloud, dans ce modèle, les données collectées par les capteurs envoient à des plateformes de services via un réseau.

- La communication des objets vers une passerelle; ce modèle est base̋ sur un intermédiaire qui fait le lien entre les capteurs et les applications dans le cloud.

- Des objets au partage des données en back-end ${ }^{12}$, l'objectif de ce modèle permet la partage des données entre les fournisseurs de services. Il est basé sur le concept «web programmable ». Les fabricants mettent en place une API permettant l'exploitation des données agrégées par d'autres fabricants [Ibid].

D'autres organismes proposent d'autres types d'architectures pour 1'IdO qui privilégient les contextes des applications. L'organisme de standardisation IEEE Standards Association (IEEE-SA) a créé le groupe de travail IEEE P2413 $3^{13}$ qui prend en compte les variétés des contextes des domaines d'applications de l'IdO. IEEE P2413 s'est fixé les objectifs suivants :

- Proposer un modèle de référence qui prend en compte les relations, les interactions et les éléments d'architectures communes pour divers domaines ;

\footnotetext{
${ }^{8}$ Titre "Microsoft Azure loTServices, architectures, demos" Téléchargeable sur le site https://www.fusionlabs.fr/language/fr/livresblancs/

${ }^{9}$ IAB a pour objectif de veiller au développement d'Internet. L'organisme est divisé en groupes de travail, des « Task forces », on retrouve notamment l'IETF (Internet Engineering Task Force).

${ }^{10}$ Les Requests For Comments (RFC), sont une série numérotée de documents officiels décrivant les aspects techniques d'Internet, ou de différents matériels informatiques.

11 "RFC 7452 - Architectural Considerations in Smart Object Networking ", consulté le 11 juin 2017 , https://tools.ietf.org/html/rfc7452.

12 Le terme "back-end » fait référence à la partie non visible d'un logiciel. II s'agit des algorithmes et autres traitements informatiques.

13 "IEEE SA - P2413 - Standard for an Architectural Framework for the Internet of Things (IoT) ", consulté le 11 juin 2017, https://standards.ieee.org/develop/project/2413.html. 
- Développer une architecture de référence qui soit comptable et prendre en compte tous les domaines des applications [Ibid].

Il propose un modèle à trois niveaux ${ }^{14}$ :

$-1^{\mathrm{er}}:$ Applications, concerne les applications et les services proposés aux clients.

- $2^{\text {ème }}$ : Cloud Computing, concerne les plateformes de services à qui sont destinées les données. Ce niveau permet d'établir de lien entre les capteurs et le réseau de plateformes et le logiciel de traitement de données [Ibid].

- $3^{\text {ème }}$ : Réseaux capteurs, le niveau le plus bas correspond aux capteurs et à la communication entre eux (le Machine-to-Machine). Il s'agit d'un réseau de capteurs qui génèrent les données et par la suite ils vont alimenter les offres de services [ROX 2017].

Ce modèle est dit «cloud-centric $»^{15}$ parce qu'il basé, en grande partie, sur le cloud. L'IEEE considère le cloud computing comme élément central pour le développement de l'IdO.

D'autres entreprises ont proposé des architectures de couches superposées comme l'entreprise américaine Cisco. En octobre 2013, Jim Green présente « Building the Internet of Things », le modèle envisagé par son entreprise pour l'IdO. Il est composé de 7 couches (figure 3 ).

IOT World Forum Reference Model

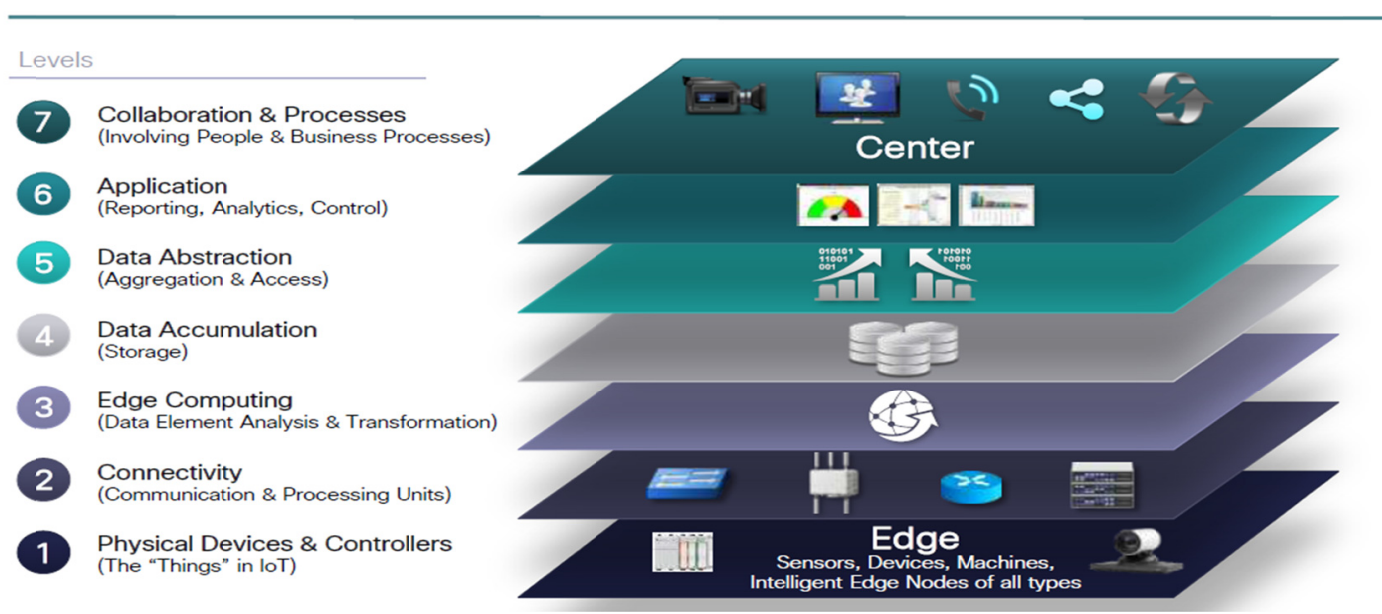

Figure 3. Les différentes couches de l'Internet des Objets (selon Cisco'16).

Ces modèles montrent l'engouement des entreprises pour les développements des écosystèmes de l'IdO ouverte et interoperable pour être acceptés par les acteurs des marchés. Malgré, ces architectures, il reste des efforts à faire pour proposer un modèle de référence globale qui prend en compte les specificités de l'IdO.

\footnotetext{
${ }^{14}$ Voir le chapitre d'Ioan Roxin et Aymeric Bouchereau, "Introduction aux technologies de l'écosystème de l'internet des objets», dans l'ouvrage Internet des objets, évolutions et innovattions, Dir. Nasreddine Bouhaï et Imad Saleh, ISTE, 2017.

15 "Cloud-centric » désigne un concept centré sur l'informatique en nuage.

${ }^{16}$ " Building the Internet of Things | An loT Reference Model », 12:31:19 UTC, http://fr.slideshare.net/Cisco/building-the-internetof-things-an-iot-reference-model. 


\section{IdO vers l'internet of Everything (IoE)}

D’après la société Cisco $^{17}$ [CIS 2013], la convergence entre les réseaux des personnes, des processus, des données et des objets, l'IdO va vers l'internet of Everything (IoE), ou « Internet du Tout connecté » (figure 4). C'est un Internet multidimensionnel qui combine les champs de l'IdO et du Big data [MON 2015].

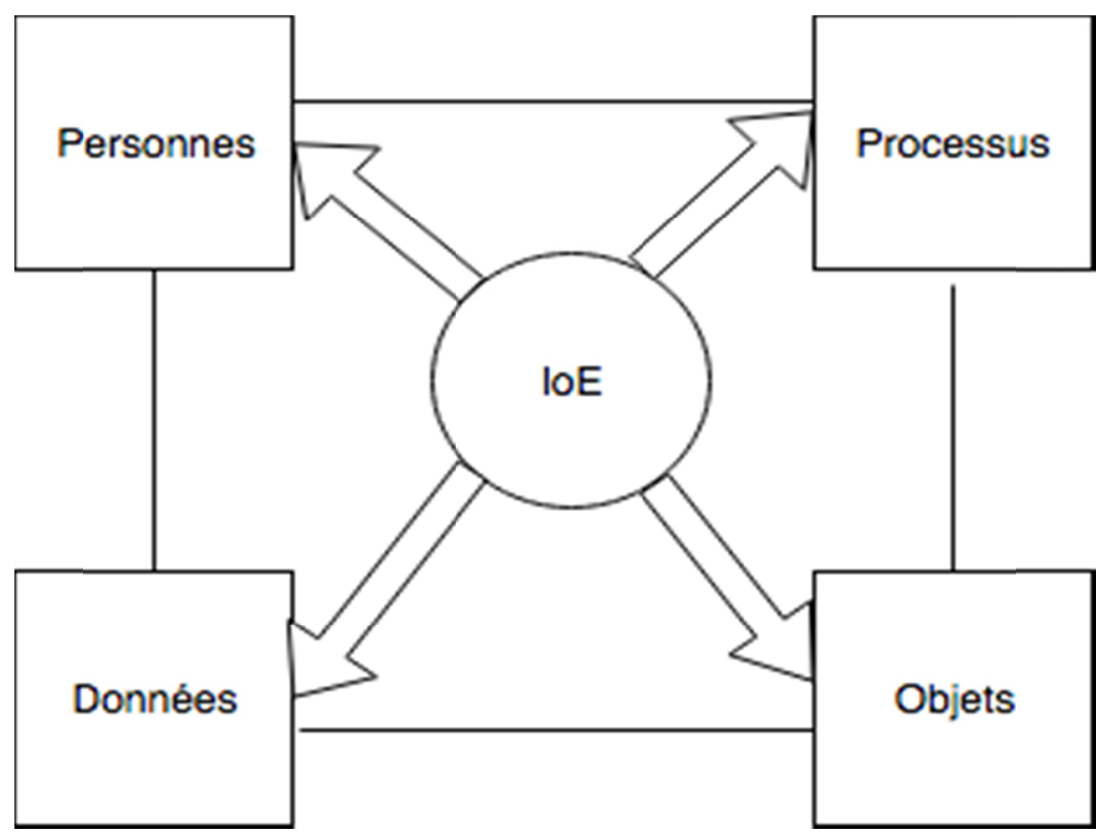

Figure 4. Internet of Everything (loE)

«Personnes: Connexion des personnes de manière plus pertinente et avec davantage de valeur.

Processus : Fournir la bonne information à la bonne personne (ou à la machine) au bon moment.

Données : S'appuyer sur les données pour faire ressortir les informations les plus utiles à la prise de décision.

Objets: Dispositifs physiques et objets connectés à l'Internet pour une prise de décision intelligente. » [Source CIS 2013, tiré de MON 2015].

IoE présente une vision plus large de l'IdO, le réseau est distribué et décentralisé, il est doté de l'intelligence artificielle à tous les niveaux pour mieux protéger les réseaux et permettre à l'usage d'avoir des données personnalisées et l'aide à prendre des decisions, c'est une vision de marketing de l'IoE.

\section{IdO et Big Data}

Le Big Data ${ }^{18}$ est un volume gigantesque de données numériques produits par les usagers d'Internet, des objets connectés, etc. Le Big Data est au coeur du développement de 1'IdO, sans les données, les OC restent des appareils physiques sans lien avec le monde réel. Le Big data est un concept global qui renvoie à 6 variables (les 6V) [ROX 2017, p. 48] :

- Volume, concerne le volume de données générées ;

\footnotetext{
${ }^{17}$ http://www.cisco.com/c/dam/en_us/solutions/industries/docs/gov/everything-for-cities.pdf

18 "Selon les archives de la bibliothèque numérique de l'ACM (Association for Computing Machinery), l'expression « Big data » serait apparue en octobre 1997, dans des articles scientifiques sur les défis technologiques à relever pour visualiser les " grands ensembles de données » [ROX 2017, p. 48] 
- Variété, concerne les types de données : données brutes, semi-structurées ou non structurées, provenant de plusieurs sources comme le web, les objets connectés, réseaux, etc. etc.

- Vitesse ou vélocité, concerne la fréquence de données générées, capturées et partagées ;

- Véracité, concerne la fiabilité et la crédibilité des données collectées ;

- Valeur, concerne le profit tiré de l'usage du Big Data ;

- Visualisation, concerne la restitution des informations pour qu'elles soient compréhensibles et interprétables malgré leur volume, leur structure, leur source et leur évolution permanente [ROX 2017].

Aujourd'hui, on sait où, quand, qui produit les données structurées ou non (Big Data). Ces précisions font "le succès des twitter qui, en plus du contenu textuel du message, encapsule des informations spatio-temporelles et par croisement avec le profil de l'auteur, ajoutent des informations sur un réseau social particulier"[SZO 2012]. Cela pose des problèmes épistémologiques et méthodologiques importants [BOY 2014, RIE 2012] concernant la multiplication des " micro-interprétations » des données et à leur representation [SZO 2012].

Ces données jouent un rôle important pour le développement économique des entreprises. Les analyses des «traces numériques » laissées par l'usage d'internet, permettent de personnaliser le service adapté au profil de l'utilisateur, mais aussi, adapté à l'endroit où se trouve l'usager. Les données produites par les objets connectés peuvent fournir des informations sur les habitudes, les compétences ou les relations des usagers. Les entreprises numériques ont déjà compris l'importance de contrôler les traces des usagers et d'en accroître leur nombre. Certaines entreprises proposent de contrôler ces données et d'identifier les objets. Ceci soulève des problèmes d'éthique et des problèmes d'authenticité pour l'usager des données produites. Dans ce contexte, plus les objets sont dotés d'algorithmes intelligents pour percevoir et agir, plus ils deviennent autonomes, ils augmentent les problèmes liés à la vie privée. Il devient donc important de développer des technologies pour permettre aux objets une auto-immunité contre les codes malveillants ou des pénétrations non autorisées pour éviter la propagation de données ou de codes erronées. Les données peuvent être localisées et stockées dans une base de données centralisée ou globalisée, dans des bases de données distribuées grâce aux technologies du cloud computing.

Certaines données peuvent être utiles ou non, mais le grand défi se situe au niveau de la contextualisation de ces données pour leur donner un sens pour créer de la valeur pour les usagers et les entreprises. Les données transmises par l'IdO sont des matériaux bruts et non traités, celles-ci sont utiles lorsque qu'elles sont combinées et traitées pour former des informations qui ont un sens. Il est nécessaire de filtrer les données pour connaître leur utilité en sachant que des données peuvent être utiles pour certains processus mais elles ne le sont pas pour d'autres. Les rôles des algorithmes intelligents peuvent venir au secours pour filtrer les pertinences des données. Néanmoins, il est donc difficile de transformer systématiquement cette immensité de données en informations et par la suite en connaissances utilisables dans la vie quotidienne. Une approche consiste à enrichir sémantiquement ces données à travers des ontologies pour faciliter leur réutilisation et permettre la mise en œuvre des mécanismes de raisonnement [SEY et al. 2015]. De plus si les connaissances sont complétées par les expériences alors les données sont transformées en savoirs et enrichissent les compétences de l'écosystème de l'IdO (figure 5). Les données générées ou produites par l'IdO soulèvent la question concernant leurs propriétaires et le droit sur elles. 


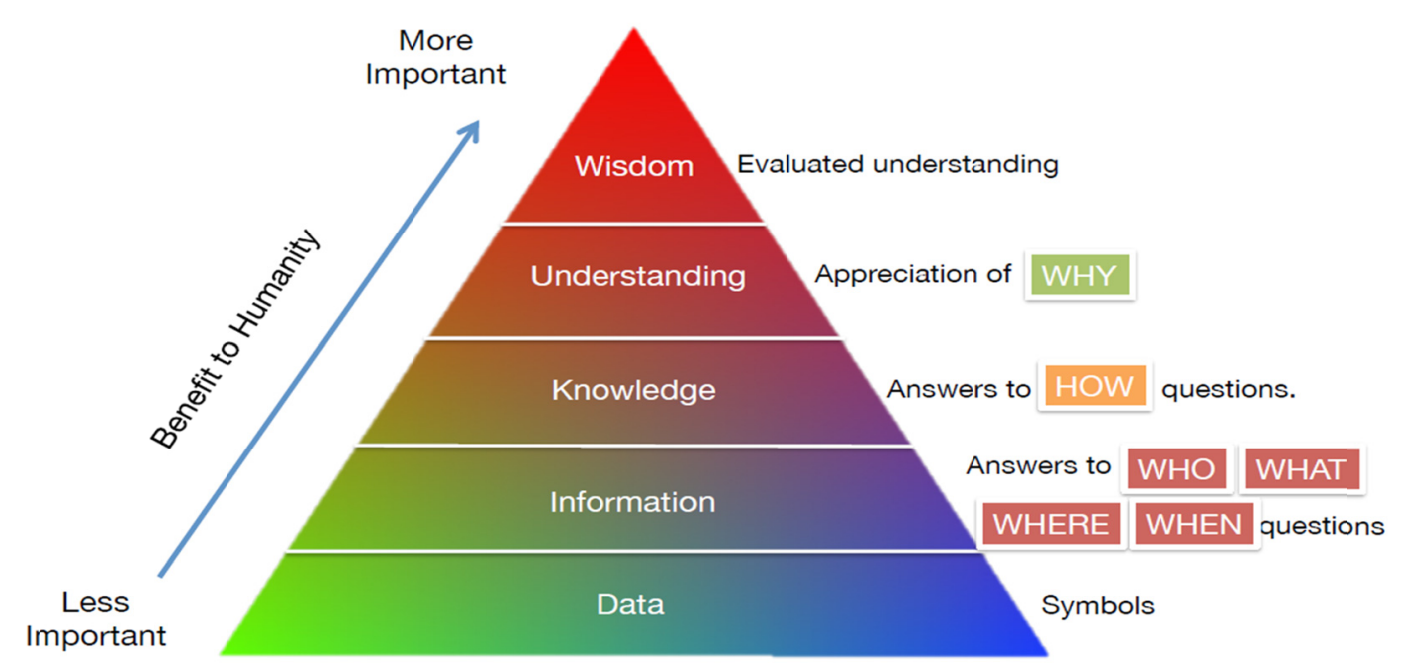

Figure 5. Slide p. 11, Tiré de la présentation de Bob Gill, P.Eng., FEC, smIEEE, 29 Novembre 2016.

\section{Cloud computing appliqué au Big Data et à l'IdO}

Les plateformes de cloud computing (informatique en nuage) vont stocker, extraire et analyser les données depuis des serveurs distants. «Le cloud computing appliqué au Big Data et à l'IdO permet la centralisation des données et des puissances de calculs. L'analyse cognitive et les techniques d'apprentissages automatiques [machine learning] font partie des " outils 》 qui permettent l'exploitation de grands volumes de données » [ROX 2017, p. 49]. Les recherches dans le domaine cognitif et l'amélioration des techniques d'apprentissages contribuent obligatoirement à l'élaboration de l'IdO et à l'exploitation au mieux des données générés par les objets. (Ibidl, p. 49)

\section{Data Science et IdO}

La Data Science ${ }^{19}$ est une science sur les données qui peut fournir une base nécessaire pour le Big Data [PIN 2017, p. 132]. Elle permet de rendre les données générées en permanence par l'Ido visuel et interprétable.

Elle est basée sur des techniques telles que le data mining, machine learning, visual analytics, le cloud, le parallel computing et la récupération de l'information [PIN 2017, MAS 2015]. Elle s'intéresse au traitement des données et au processus de visualisation dans tous les domaines basés sur le flux permanent des données tels que le transport, l'assurance, la santé, etc. [PIN 2017].

La data science permet de définir avec précision les formes actuelles de visualisation analytique [PIN 2017]. La visualisation des données est une étape fondamentale pour identifier les modèles, les tendances et les relations entre les données en utilisant le Big Data. La visualisation permet l'émergence du domaine d'étude dit le visual analytics qui est basé sur "l'utilisation et l'analyse visuelle interactive de données volumineuses et complexes (dataset)" [Ibid]. Il "représente le processus analytique et requiert un haut degré de surveillance et d'interaction homme-machine" [PIN 2017, p. 132]. La Data Science permet de découvrir de nouveaux algorithmes pour la visualisation des données [CHE 2015].

La figure 6 résume le rapport entre les technologies décrites ci-dessous et l'IdO [NOY 2017].

\footnotetext{
${ }^{19}$ Voir le chapitre de Pinto A.L. et al « la visualisation de l'information pour l'internet des objets » dans l'ouvrage « Internet des objets, évolutions et innovations », Dir. Nasreddine Bouhaï et Imad Saleh, ISTE 2017.
} 


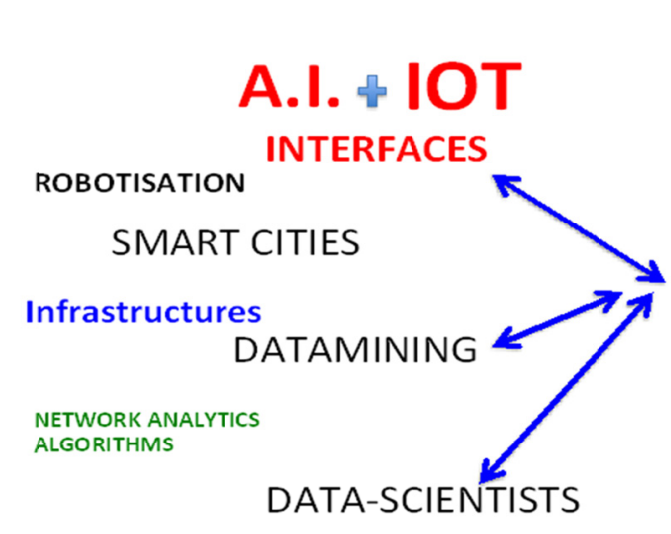

SANTE

AGRICULTURE

TRANSPORT

EDUCATION

ENERGIES

EAU...

\section{BIG DATA}

\section{MILITAIRE}

URBANIZATION

CONSUMERISME

ENTERTAINMENT

IMMERSIONS...

Figure 6. Tirée de l'article de Jean-Max Noyer [NOY 2017].

\section{Les enjeux et les défis de l'IdO}

L'IdO touche à tous les domaines de la co-construction des savoirs et des connaissances, de la gestion des entreprises et des administrations en passant par les technologies éducatives et hyperUrbaines, il transforme notre relation localisée en relation globalisée en dépassant les frontières géographiques [SAL 2012, 2014]. Ces bouleversements à la fois technologiques, sociétaux et humains soulèvent de nombreuses questions concernant la mutation sociétale et économique mais également pour la recherche, la créativité et l'innovation. Il est important de penser à construire de nouveaux instruments pour un devenir technique au service du développement humain.

\subsection{Defis Technologiques}

L'IdO est au centre de tous les développements technologiques modernes où la question des interfaces, des protocoles dans une situation stratégique majeure, est posée. "Toute information, qu'elle soit textuelle, sonore ou visuelle [mais aussi gestuelle] est en interaction permanente avec l'ensemble des autres, elle devient réticulaire ${ }^{20}$ ". L'IdO transforme internet en HyperRéseau mondialisé dont tous les points formeraient autant de nœuds d'information possible sans frontier [Ibid].

Les enjeux en terme de programmation informatique sont immenses. De nouvelles méthodes de conception, de développement, de débogage et de maintenance doivent voir le jour. A partir de langages symboliques innovants, les informaticiens de demain transformeront de simples lignes de code en agents autonomes capables d'exister dans la complexité des systèmes ubiquitaires et distribués, pour s'adapter aux demandes des utilisateurs, afin de recommander de nouveaux usages voir même de les accompagner dans l'évolution de leurs modes d'existence.

Un défi majeur pour l'IdO à la fois technique et utile, c'est de pouvoir gérer l'hétérogénéité technologique et des normes d'objets couplées à des multitudes de besoin d'applications et des usages en terme de services de sécurité. Sachant que ces besoins peuvent évoluer dans le temps selon le contexte et le goût. En effet, comment assurer l'authentification individuelle de plusieurs millions d'objets hétérogènes, dotés des technologies de communication hétérogènes, à travers des domaines administratifs multiples ? [VER 2011]. Ceci soulève des problèmes de gestion et de sécurité des objets dans un milieu hétérogène au niveau physique et logique. "En effet, l'Internet des objets est un système complexe dans lequel interagissent des personnes avec un écosystème technologique à base d'objets intelligents à travers des processus complexes. Les interactions de ces quatre composantes de l'IdO (personnes, objets intelligents, écosystème technologiques, processus) font émerger une

\footnotetext{
${ }^{20}$ Jean-Pierre Balpe, http://hyperfiction.blogs.liberation.fr/2007/10/25/des-hypertextes/, consulté le 10 janvier 2018
} 
dimension systémique à la sécurité de l'IdO» [CHA 2012, p. 54]. Par la suite, les tensions sur la sécurité de l'IdO sont créées lors de l'interaction des objets intelligents avec son environnement. Ces tensions (confiance, responsabilité, identification et auto-immunité) sont caractérisées par une dimension cognitive et systémique induite par l'autonomie grandissante des objets [CHA 2012].

\subsection{Défis Sociétaux}

L'enjeu du futur est de répondre de manière aussi créative que possible, aux inquiétudes anthropologiques, aux changements de régime climatique, de la biodiversité, aux implications industrielles et à la transition énergétique, aux implication bio-politiques ainsi qu'éthiques d'accessibilité, de diversité culturelle, de redéfinitions de la notion de «données personnelles ». Mais c'est aussi poser la question de la dissémination des technologies intellectuelles comme contrepouvoirs dans une société datacentrique, en proposant des modèles d'analyses, des cadres conceptuels à la hauteur de ce qui s'avance, des méthodes de conception et des règles d'usages reconnus par l'ensemble des usagers, par les nouvelles intelligences collectives d'usages.

\subsection{Défis environnementaux}

L'augmentation sans cesse des objets connectés a des conséquences sur l'environnement. Elle peuvent être traduites par l'augmentation d'une part de déchets électroniques et leurs recyclages et d'autre part sur la consommation de l'énergie. C'est un défi majeur qui attire de plus en plus de gouvernements pour étudier l'impact des objets connectés sur le réchauffement climatique et leurs conséquences sur les populations. Par exemple: L'Union Européenne ${ }^{21}$ a fixé comme objectif pour l'année 2020 de réduire de $20 \%$ les émissions de CO2 et d'améliorer l'efficacité énergétique de $20 \%$ pour atteindre $20 \%$ d'énergie renouvelable. Il est donc nécessaire que les entreprises étudient avant la fabrication des OC leurs durées de vie et leur utilité pour les usagers afin de diminuer des fabrications d'objets non nécessaires et avec une consommation d'énergie inutile.

\subsection{Confiance dans I'ldO}

Ainsi, le degré de confiance en l'IdO et son acceptation sont des conditions primordiales pour la mise en place de mesures adéquates de protection des données personnelles et de la vie privée. En effet, il est important de prévoir lors de la conception des éléments de l'IdO, l'ensemble des exigences des usagers et la sécurité des données [COM 2009]. Ils existent des solutions basées sur la cryptographie ou sur la gestion de clés à base de pré-distribution qui pourraient accommoder les contraintes de ressources des objets [CHA 2012]. Néanmoins, des questions sont soulevés sur la robustesse et la solidité de telles solutions à un IdO comportant potentiellement des millions d'objets.

\subsection{Defis pour les entreprises}

L'IdO croît sans cesse : «la mémoire invisible» du web e basée sur les traces laissées par les utilisateurs enregistrées dans des bases de données et analysées par le biais de méthodes statistiques et autres. Il nous semble qu'il est important que les acteurs publics ou privés saisissent l'opportunité de l'évolution de l'IdO et de cette mémoire pour proposer des approches originales pour bien être de l'humanité.

- Pour les entreprises, il s'agit de comprendre les comportements des usagers et les tendances des consommateurs pour proposer des services et des produits adaptés.

- Pour les acteurs politiques, ils peuvent suivre les tendances des sociétés pour ajuster leurs modes d'organisation, et leurs valeurs, adaptant leurs programmes et leur mode de fonctionnement.

\footnotetext{
${ }^{21}$ Site : https://ec.europa.eu/clima/policies/strategies/2020 fr (consulté le 4 Février 2018). 


\subsection{Défis pour les chercheurs}

- Pour les chercheurs en Sciences Humaines et Sociales (SHS), ils disposent de moyens d'observation fantastiques « totaux » qui promettent "de révolutionner les méthodologies classiques en effaçant le clivage entre micro et macro, entre qualitatif et quantitative” [RIE 2010].

- Pour les chercheurs en Sciences (informatique, mathématiques, etc.), ils disposent d'une quantité de données pour tester la fiabilité, la rapidité et l'invention des algorithmes.

- Pour les chercheurs en IHM, ils disposent de données et d'outils qui leur permettent d'imaginer et de créer des artefacts esthétiques et compréhensibles pour visualiser des données arrivées de manière quasi-continue [PIN 2017].

«La quantité et la richesse des données laisseraient penser que l'on peut passer, par une sorte de « zoom », de l'ensemble à l'individu et de la moyenne à l'idiosyncrasie » [RIE 2010]. Les traitements et l'analyse des traces numériques et des données produites par l'IdO sont en train de changer à la fois les modes de production et de communication de connaissances par les chercheurs mais également l'organisation de la recherche, son économie et son rôle dans la société [RIE 2010].

L'IdO soulève une quantité de problèmes épistémologiques et sociotechniques : liberté-contrôle, autorité-indépendance, associativité-unicité, automatisme-maîtrise, actions-interactions, contextualisation-décontextualisation, adaptativité-intégrité, etc. Nous devrons désormais apprendre à « penser à la fois le mobile, le flou, l'incertain, le proche et le lointain ${ }^{22}$ ».

\section{Opportunités et menaces dans l'écosystème de l'IdO}

Nous pouvons constater que les applications et services développés autour de l'IdO sont susceptibles d'améliorer la vie quotidienne, en optimisant et automatisant certaines activités, en revanche des interrogations accompagnent l'IdO concernant les usages et les contrôles des informations afin d'éviter le piratage, le surveillance abusive des usagers, etc. Dans le tableau 2, nous ferons une synthèse sur les opportunités et les menaces qui représentent les développements de l'IdO au niveau technique, humain et socio-économique.

\footnotetext{
22 Jean-Pierre Balpe, « Des hypertextes à I'hypermonde » (http://nt2.uqam.ca/fr/actualites/des-hypertextes-l'hypermonde, consulté le 6/12/2016). 


\begin{tabular}{|c|c|}
\hline Marché en pleine d'expansion & Accès au marché difficile pour les petites entreprises \\
\hline Diminution du coût des technologies & Fragilité physique des objets \\
\hline Compétitivité qui favorise l'innovation & $\begin{array}{c}\text { Influencer sur la prise de décision pour orienter } \\
\text { l'innovation }\end{array}$ \\
\hline Evolution technologique (Ipv6, \\
miniaturisation)
\end{tabular}

Tableau 2. Opportunités et menaces

\section{Sécurité de l'IdO}

Vue l'émergence des technologies de l'IdO dans tous les domaines de la vie quotidienne, cela pose question sur la sécurité l'IdO. Elle doit être pensée sous 3 angles complémentaires : technologique, humain et systémique (CHA 2012) :

- La protection de la technologie concerne la sécurité des données, des communications et des infrastructures réseaux et leurs fonctionnalités.

- La protection des personnes concerne la protection de la vie privée des usagers («privacy») pour éviter des litiges causés éventuellement par l'IdO.

- «La protection des systèmes interconnectés et hébergeant les objets de l'IdO, concernera la protection des objets eux-mêmes livrés à ces systèmes et les processus qu'ils contrôleront » (Ibid, p. 62)

Yacine Challal (CHA 2012) a analysé les travaux existants et les besoins de l'IdO en terme de sécurité, il conclut que les développements potentiels se déroulent autour de trois axes à court, moyen et long terme. Dans le tableau 3, il illustre ces trois axes et synthétise les verrous scientifiques et technologiques derrière chacun. 


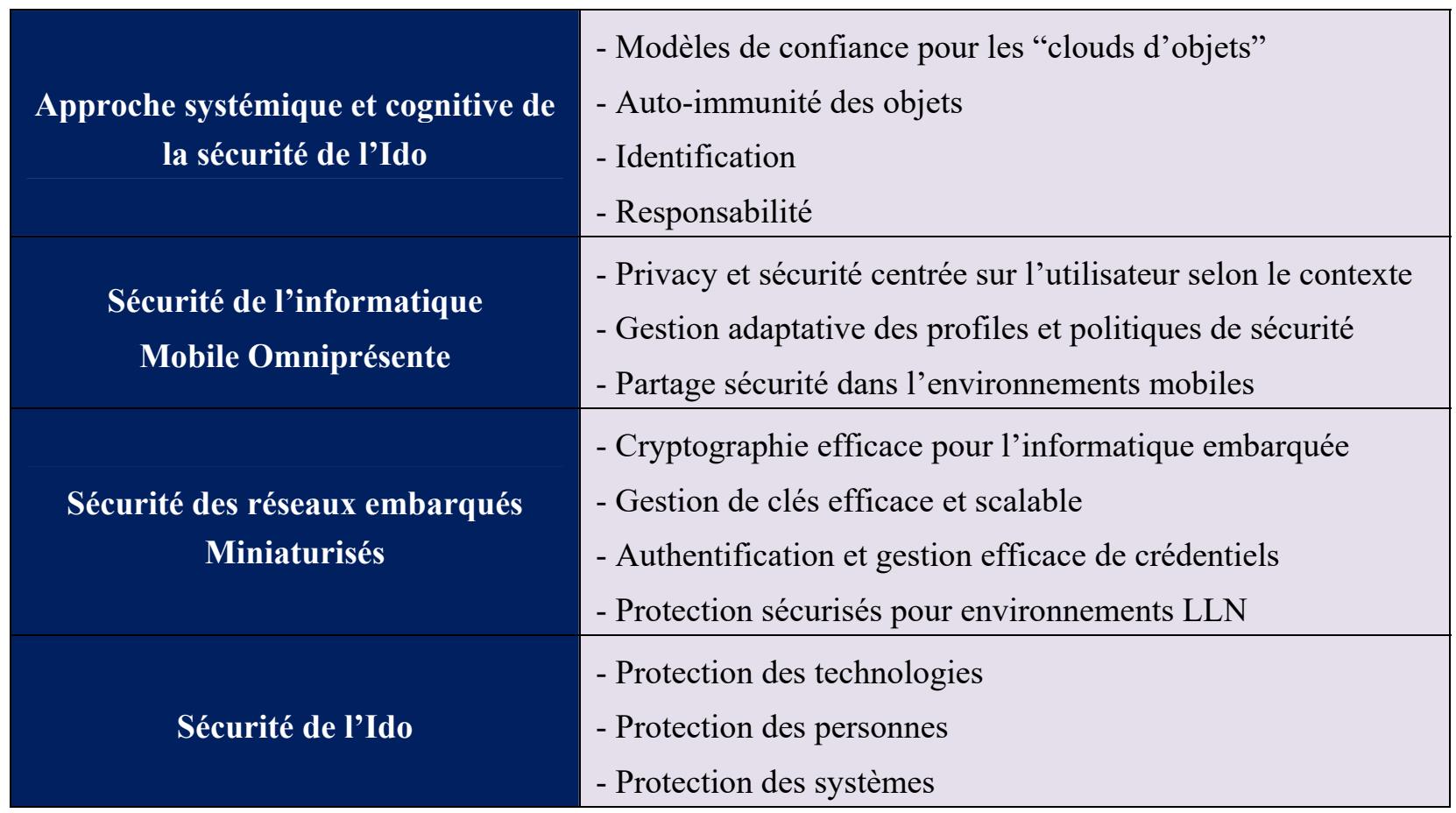

Tableau 3. Trois grands chantiers pour la sécurité et la privacy dans I'ldO (HDR Yacine Challal 2012, p. 63, nous avons modifié seulement la présentation)

\section{Blockchain et IdO}

\subsection{Définition}

La Blockchain est une technologie développée pour la cryptomonnaie Bitcoin par par Satoshi Nakamoto (pseudonymme) en 2008 [NAK 2008]. La société Blockchain France a définie "La blockchain est une technologie de stockage et de transmission d'informations, transparente, sécurisée, et fonctionnant sans organe central de contrôle. Par extension, une blockchain constitue une base de données qui contient l'historique de tous les échanges effectués entre ses utilisateurs depuis sa création. Cette base de données est sécurisée et distribuée : elle est partagée par ses différents utilisateurs, sans intermédiaire, ce qui permet à chacun de vérifier la validité de la chaîne ${ }^{23}$ ».

La Blockchain ${ }^{24}$ est un système de registre distribué où l'enregistrement des transactions s'effectue à travers des nœuds multiples dans un réseau pair à pair $(\mathrm{P} 2 \mathrm{P})$.

De «Commotion» http://commotionwireless.net/ à la «Blockchain»: variations autour des systèmes décentralisés. Il s'agit de s'affranchir d'une instance centrale en mettant en place des dispositifs qui fonctionnent indépendamment d'une instance centrale. Avec tous les problèmes politiques qui vont avec dans le cadre d'une société d'hypercontrôle. ${ }^{25}$

\subsection{Fonctionnement}

"Toute Blockchain publique fonctionne nécessairement avec une monnaie ou un token (jeton) programmable ${ }^{26} »$, Bitcoin en est un exemple. Les utilisateurs du Bitcoin, qui se connaissent par l'échange de leurs clés publiques, génèrent et diffusent des transactions sur le réseau pour transférer de l'argent. Ces transactions sont regroupées dans un bloc par les utilisateurs. Lorsqu'un bloc est rempli,

\footnotetext{
${ }^{23}$ Tiré du site de la société : https://blockchainfrance.net/decouvrir-la-blockchain/c-est-quoi-la-blockchain/, consulté le 30 Janvier 2018.

${ }^{24}$ Voir le blog Ahmed Banafa: https://ahmedbanafa.blogspot.com/ pour plus amples d'informations, consulté le 30 Janvier 2018.

${ }^{25}$ S'affranchir de l'autorité centrale avec la blockchain Serge Abiteboul « La Recherche » Novembre 2017.

${ }^{26}$ Site de la socité blockchain, : https://blockchainfrance.net/decouvrir-la-blockchain/c-est-quoi-la-blockchain/, consulté le 30 Janvier 2018.

(c) 2018 ISTE OpenScience - Published by ISTE Ltd. London, UK - openscience.fr 
celui-ci est ajouté à la Blockchain après un processus de minage. Pour miner un bloc, des nœuds du réseau, appelés "mineurs", essayent de résoudre un problème cryptographique appelé "Proof-ofWork (PoW)» ou preuve de travail. "Une fois le bloc validé, il est horodaté et ajouté à la Blockchain ${ }^{27} \gg$. À ce moment, la transaction est visible pour le récepteur ainsi que sur l'ensemble du réseau (Figure 7).

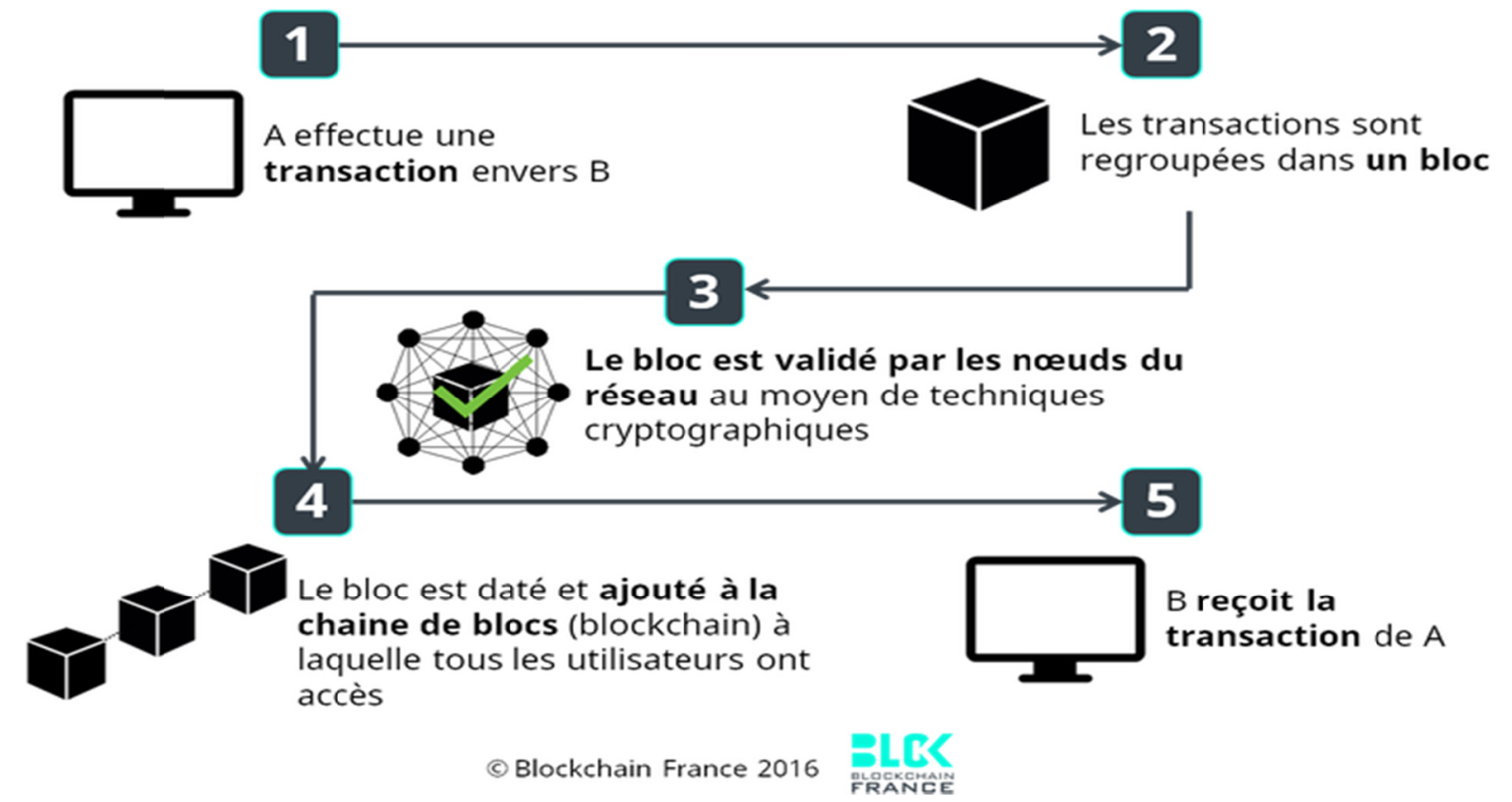

Figure 7. tirée du site de la société :

https://blockchainfrance.net/decouvrir-la-blockchain/c-est-quoi-la-lblockchain/

Dans le contexte de l'Internet des Objets, du fait de l'interconnexion de systèmes hétérogènes qui génèrent un volume important de données personnelles, notre société numérique se trouve face à plusieurs défis (voir ci-dessous). Vu le fonctionnement de blockchain distribué et ses mécanismes de consensus permettant de concilier des intérêts divergents et une confiance répartie grâce à la suppression du tiers de confiance unique, elle peut offrir des réponses à certains défis.

Cependant, comme énoncé par Dorri et al. [DOR et al 2016], l'adaptation de cette technologie à l'IdO n'est pas simple. En effet, le processus de minage requiert une grande capacité de calcul alors que la majorité des appareils de l'IdO présentent des ressources limitées. Aussi, ce processus prend du temps, alors que, dans la plupart des applications IdO, une faible latence est souhaitable. De plus, la Blockchain devient difficile à utiliser à mesure que le nombre de nœuds dans le réseau augmente, tandis que les réseaux IdO devraient contenir un grand nombre de nœuds. Enfin, les protocoles sousjacents de la Blockchain créent un trafic indirect important, ceci il peut pose des problèmes pour certains dispositifs IdO à bande passante limitée [DOR et al 2016].

\subsection{Applications}

Dans ce cadre, de nombreux chercheurs se sont penchés sur l'utilisation et l'adaptation de la Blockchain dans l'IdO. Parmi eux, Mettler [MET 2016] qui illustre les utilisations de cette technologie dans le domaine de la santé, que ce soit pour la gestion de santé publique, la recherche médicale basée sur les données personnelles des patients ou pour l'assurance qualité dans la production de médicaments. Aussi, Dorri et al. [DOR et al. 2017] ont présenté une adaptation de la Blockchain pour le cas de maisons intelligentes. En regroupant les appareils en clusters et en rajoutant des Blockchains

\footnotetext{
${ }^{27}$ ibid, site de la socité Blckchain, : https://blockchainfrance.net/decouvrir-la-blockchain/c-est-quoii-la-blockchain/, consulté le 30 Janvier 2018. 
locaules, ils montrent qu'il est possible de réduire la charge sur le réseau tout en garantissant la sécurité des données des utilisateurs et la protection de leurs vies privées. Enfin, dans leur article [CHR 2016], Christidis et al. exposent l'utilisation de la technologie Blockchain pour les « smart contracts » ou contrats intelligents. Ces derniers sont des "programmes autonomes qui exécutent automatiquement les conditions et termes d'un contrat, sans nécessiter d'intervention humaine une fois démarrés ${ }^{28} \gg$. Les contrats intelligents peuvent être intéressants pour l'IdO car ils permettent l'automatisation de longs processus tout en assurant leurs vérifiabilités.

L'intégration de la Blockchain dans l'IdO entraînera des transformations significatives dans plusieurs secteurs, conduisant à de nouveaux modèles et nous demandant de reconsidérer la manière dont les systèmes et les processus existants sont mis en œuvre. La Blockchain peut aussi être un moyen d'assurer la sécurité des données des utilisateurs ainsi que la protection de la vie privée, permettant ainsi une plus grande adoption de $\mathrm{l}^{\prime} \mathrm{IdO}^{29}$.

\section{Conclusion}

Dans un monde « " hyperconnecté » via des objets connectés où les usagers sont à la fois émetteurs et récepteurs des données, l'IdO ouvre des champs nouveaux à explorer pour les sciences de l'information et de la communication pour étudier d'une part les enjeux sociétaux de ces nouveaux bouleversements technologiques et numériques et d'autre part analyser si les objets connectées répondent à des besoins d'usagers de plus en plus exigeants en matière de service, de communication et d'information. L'Internet des objets doit être traiter sous deux aspects d'une part de la réalité industrielle et technologique des objets connectés comme la gestion de l'entreprise, l'e-administration, l'e-gouvernement mais aussi gestuelle : podomètre, direction du regard, GPS, etc. et d'autre part les impacts des objets connectés dans la vie quotidienne dans les domaines suivant: la santé, l'habitat, l'automobile, l'assurance, etc.

La figure 8 illustre à notre avis les développements futurs de l'IdO.

\footnotetext{
${ }^{28}$ :Site de la société : https://blockchainfrance.net/decouvrir-la-blockchain/c-est-quoi-la-blockchain/, consulté le 30 Janvier 2018.

${ }^{29}$ Cette partie a été rédigée en collaboration avec le doctorant Amri Toumia, janvier 2018.
} 


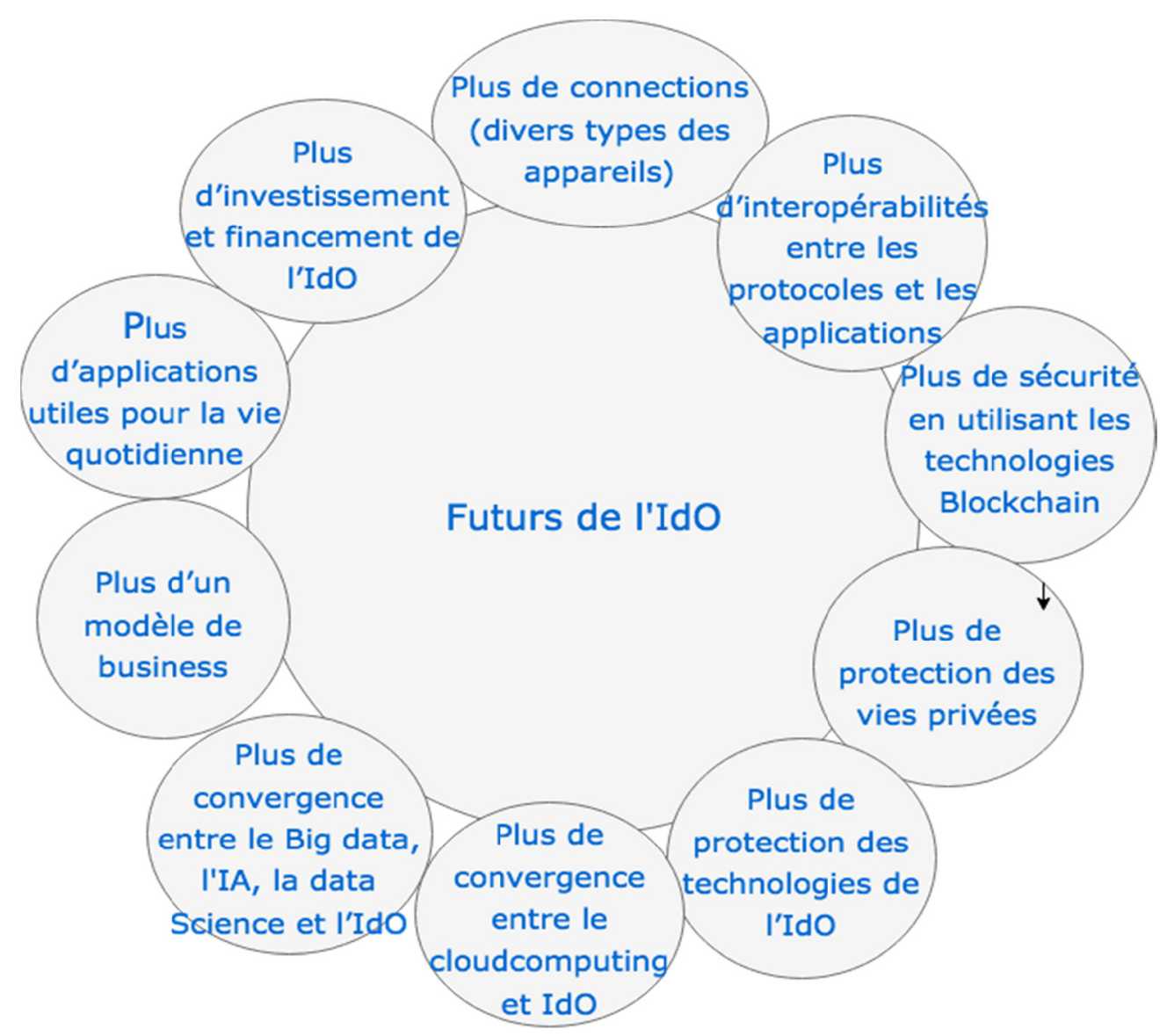

Figure 8. Futurs de l'ldO

\section{La bibliographie}

[AMR 2017] AMROUM H., TEMKIT, M'H., AMMI H. Ammi, M., “Apprentissage en profondeur des données brutes de l'activité humaine", Revue : Internet des objets, Numéro : Numéro 2, Volume : 2, Date : 2017/06/19, DOI : 10.21494/ISTE.OP.2017.0150, ISSN : 2514-8273

[BOU 2017] BOUHAÏ N., "Internte des objets : des objets envahissantes ou indisponsables", dans Bouhaï N. et Saleh I., (dir.) "Internet des objets : Evolutions et Innovations", ISTE Editions Londres, Mai 2017.

[BOY 2012] BOYD D., CTAWFORD K., Critical Question for Big Data, article consulté le 2/02/2017 site : https://people.cs.kuleuven.be/ bettina.berendt/teaching/ViennaDH15/boyd_crawford_2012.pdf

[CHA 2012] CHALLAL Y., "Sécurité de l'Internet des Objets : vers une approche cognitive et systémique”, HDR, Juin 2012, UTC.

[CIS 2013] Cisco, "Value of the Internet of Everything for Cities", States ans Countries, 2013.

[CLU 2010] Cluster of European Research Projects on the Internet of Things, Vision and Challenges for Realising the Internet of Things, March 2010.

[COM 2009] Commission des Communautés Européennes, L'Internet des Objets : un plan d'action pour l'Europe, 2009.

[CRO 2017] CROUZY S., BORKOWSKI S., COQUILLART S., "Ambient Atoms\&nbsp;: un périphérique pour la visualisation ambiante d'informations", Internet des objets , Numéro : Numéro 2, Volume : 2, Date : 2017/06/19, DOI : 10.21494/ISTE.OP.2017.0152 ISSN : 2514-8273

[CHE 2015] Chen, L. M.; Su, Z; Jiang, B. (2015) "Mathematical Problems in Data Science: theoretical and practical methods”, Amsterdam. Springer International Publishing Switzerland.

[CHR 2016] CHRISTIDIS K. and DEVETSIKIOTIS M., "Blockchains and smart contracts for the internet of things," IEEE Access, 2016.

[DOR 2016] DORRI A., KANHERE S.S., and JURDAK R., "Blockchain in internet of things: Challenges and Solutions," Aug. 2016. 
[DOR 2017] DORRI A., S. S. Kanhere, R. Jurdak, and P. Gauravaram, "Blockchain for IoT Security and Privacy: The Case Study of a Smart Home,” Ieee Percom Work. Secur. Priv. Trust Internet Thing, no. March, 2017.

[INT 2005] International Telecommunication Union, "Ubiquitous Network Societies: their impact on the telecommunication industry", ITU Workshop on Ubiquitous Network Societies, April 2005.

[GAG 2017] GAGNERE G., PLESSIET C., SOHIER R., "Espace virtuel interconnecté et Théâtre. Une recherchecréation sur l'espace de jeu théâtral à l'ère du réseau", Revue : Internet des objets, Numéro : Numéro 2, Volume : 2, Date : 2017/06/19, DOI : 10.21494/ISTE.OP.2017.0151, ISSN : 2514-8273

[GSM] GSMA http://www.gsm.org

[MAS 2015] «Master In Data Science», disponible à l'adresse : https ://www.city.ac.uk/ courses/postgraduate/data-science-msc, consulté le 6 février 2017.

[MAV 2003] MAVROMMATI I., KAMEAS A., " The evolution of objects into hyper-objects: will it be mostly harmless?», Personal and Ubiquitous Computing. 2003. Disponible sur : http://dx.doi.org/10.1007/s00779-003-0223-1

[MET 2016] METTLER M., "Blockchain technology in healthcare: The revolution starts here," in 2016 IEEE $18^{\text {th }}$ International Conference on e-Health Networking, Applications and Services (Healthcom), 2016, pp. 1-3.

[MON 2015] Institut Montaigne, "Big data et objets connectés Faire de la France un champion de la révolution numérique », Rapport Avril 2015.

[NAK 2008] NAKAMOTO S., "Bitcoin: A Peer-to-Peer Electronic Cash System,” 2008.

[NOY 2017] NOYER Jean-Max, "L'Internet des Objets, 1'Internet of Everything : quelques remarques sur l'intensification du plissement numérique du monde", Revue : Internet des objets, Numéro : Numéro 1, Volume : 1, Date : $2017 / 04 / 4$, DOI : $10.21494 /$ ISTE.OP.2017.0134, ISSN : 2514-8273

[PIN 2017] PINTO A. L., GONZALES-AGUILAR A., DUTRA M. L., SEMELER A. R. M., DENISCZWICZ, CLOSEL, C., "La visualisation de l'information pour l'Internet des objets", dans Bouhaï N. et Saleh I., (dir.) "Internet des objets : Evolutions et Innovations", ISTE Editions Londres, Mai, 2017.

[RIE 2010] REIDER, B., "Pratiques informationnelles et analyse des traces numériques : de la représentation à l'intervention", Etudes de communication, 2010, N³5, p.91-104, site : https://edc.revues.org/2249, consulté le 03/02/2017.

[RIE 2012] REIDER, B. \& RÖHLE, T. "Digital methods: five challenges", In D.M BERRY (Ed.) Understanding digital humanities. Houndmills: Palgrave Macmillan, p.67- 84.

[ROX 2017] ROXIN, I., BOUCHEREAU A., "Ecosystème de l'Internet des Objets", dans Bouhaï N. et Saleh I., (dir.) "Internet des objets : Evolutions et Innovations ”, ISTE Editions Londres, Mai 2017.

[SAL 2017] SALEH, I., 2017. « Les enjeux et les défis de l'Internet des Objets (IdO) », Revue « Internet des objets » 1. DOI:10.21494/ISTE.OP.2017.0133

[SAL 2012] SALEH I., HACHOUR H., « Le numérique comme catalyseur épistémologique », Revue Française des Sciences de l’Information et de la Communication, $\mathrm{N}^{\circ} 1,2012 \mathrm{http} / / \mathrm{rfsic}$.revues.org/168,

[SAL 2014] SALEH I., HACHOUR H. et BOUHAI N., « Les frontières numériques » L’Harmmattan, septembre 2014, 260 pages.

[SEY et al. 2015] SEYDOUX N., BEN ALAYA M., HERNANDEZ N., MONTEIL T., HAEMMERLE O,. "Sémantique et Internet des objets : d'un état de l'art à une ontologie modulaire", 26es Journées francophones d'Ingénierie des Connaissances, Jun 2015, Rennes, France. https://hal.archives-ouvertes.fr/IC-2015/hal-01166052, consulté le 3.03.2017.

[SZO 2017] SZONIECKY S., SAFIN S. « Modélisation éthique de l’Internet des Objets », Volume : 2, Date, 2017/06/19, dans la revue « Internet des objets » ISTE, DOI:10.21494/ISTE.OP.2017.0148.

[SZO 2011] SZONIECKY S., "Evaluation et conception d'un langage symbolique pour l'intelligence collective, Vers un langage allegorique pour le Web", These Soutenue le 7 decembre 2012, Université Paris 8.

[THE 2013] THEBAULT P., « La conception à l'ère de l'Internet des Objets : modèles et principes pour le design de produits aux fonctions augmentées par des applications", Thèse soutenue le 31 mai 2013, ParisTech.

[VER 2011] VERMESAN, O., et al. "Internet of Things Strategic Research Roadmap", Cluster of European Research Projects on the Internet of Things, 2011.

[WEI 1991] WeISER M., « The computer for the XXIe century », Scientific American, vol. 265, n 3, p. 3- 11, 1991. 
[WEI 1993] WEISER M., « Hot Topics : Ubiquitous Computing » IEEE Computer, octobre 1993.

[WOO 2011] WOOD L., "Today, the Internet, tomorrow-the Internet of Things", ComputerWorld,http://www.computerworld.com/s/article/9221614/Today_the_Internet_tomorrow_the_Internet_of Things, November 2011. 